\section{Genetic Factors and in Vitro Manipulations Influence Seed Dormancy in Cucumber}

\author{
Nawab Ali, Robert M. Skirvin, Walter E. Splittstoesser, \\ David E. Harry, and William L. George \\ Department of Horticulture, University of Illinois, Vegetable Crops \\ Building, Urbana, IL 61801
}

Additional index words. Cucumis sativus, tissue culture, biotechnology

\begin{abstract}
Seed lots with the genetic background of 'Baroda' and 'Marketer' cucumber (Cucumis sativus L.) containing all possible combinations (DF Df, Dfdf, dfdf) of $d f$ (which increases dormancy) and $D f$ (wild type) were used. Dormancy was not solely due to the genotype $d f d f$ and clear effects of genetic background were apparent. The $d f$ allele in the homozygous state induced a strong dormancy in 'Baroda', but the $D f$ gene could not restore normal germination. However, $D f$ did reduce the dormancy period to 85 days. In 'Marketer', $d f$ did not delay germination. Any treatment (puncturing, removal, cutting) that damaged the inner integument allowed 'Baroda' $d f d f$ to germinate, indicating an intact integument was essential for maintaining dormancy in this cultivar. All 'Baroda' $d f d f$ embryonic axes without the cotyledons germinated in 5 days. 'Baroda' $d f d f$ seeds with intact integuments imbibed adequate water to germinate but remained dormant, suggesting that the effect of the integument on dormancy was not related to imbibition.
\end{abstract}

Cucumber seeds normally germinate a few days after being extracted from freshly harvested mature fruits. However, Shifriss and George (1965) reported seed dormancy in an Indian cultivar known in the United States as 'Baroda'. Seeds remained dormant for 6 to 12 months, but dormancy could be overcome by exposing the seeds to $50 \mathrm{C}$ and $90 \%$ relative humidity for 6 days.

Whereas most cucumber cultivars are day neutral, Shifriss and George (1965) also observed that flowering in 'Baroda' was affected by photoperiod. They showed that the $d f$ allele caused delayed flowering in 'Baroda' and was recessive to the $D f$ allele for normal flowering found in other cucumber cultivars. Seed dormancy and flowering behavior in 'Baroda' were not independent characteristics. They suggested that the $D f$ locus was either linked with a gene (or several) affecting seed dormancy or the $D f$ gene interacted with other genes to affect germination.

This study was designed to determine 1) how the delayed flowering $(d f)$ gene affects seed dormancy; 2) if seed dormancy could be overcome by manipulations of the seed in vitro; and 3) whether seed dormancy is associated with specific seed tissues.

In the 1960s (Shifriss and George, 1965) the $d f$ gene was identified in 'Baroda' (BA) and transferred to the normal-flowering 'Marketer' (MK). Through backcrossing, the $D f$ gene from 'Marketer' was also put into 'Baroda' (W.L.G., unpublished). The plants were self-pollinated over many generations

Received for publication 17 Sept. 1990. The cost of publishing this paper was defrayed in part by the payment of page charges. Under postal regulations, this paper therefore must be hereby marked advertisement solely to indicate this fact. to obtain near-isogenic lines. These genotypes, homozygous for alleles $D f$ and $d f$, were used in these studies. Seeds of each genotype (MK $D f D f$, BA $D f D f$, MK $d f d f$, BA $d f d f$ ) were germinated in petri dishes; the seedlings were transferred to a glasshouse and grown at Urbana, Ill., under the natural long days of April to August. One flower on each plant was self-pollinated, and another flower on the same plant was outcrossed. Reciprocal crosses were made in all 16 pair-wise combinations. Fruits were harvested 45 days after anthesis and the seeds extracted, cleaned, and air-dried. At various times after harvest, 30 seeds of each genotype were germinated in petri dishes for 15 days.

Delayed germination in 'Baroda' was not explained completely by the $d f$ gene. $D f D f$ genotypes did not germinate normally (Table
${ }^{2} \mathrm{MK}=$ 'Marketer'; $\mathrm{BA}=$ 'Baroda'.

ySeeds remained dormant for $>144$ days.
1). If the only gene regulating dormancy in cucumber were $d f d f$, self-pollinated MK $d f d f$ (Table 1, cross 3) should have shown dormancy, but did not. BA $d f d f$ was dormant (Table 1, cross 2), but BA DfDf was also somewhat dormant (Table 1, cross 4). It is evident from cross 3 that the $d f$ gene did not cause dormancy with the MK background. These results indicate an interaction between genetic background (i.e., cultivar) and dormancy induction by the $d f$ gene. With 'Marketer' background, neither seeds homozygous for the $D f$ allele (Table 1, cross 1) nor those homozygous for $d f$ allele were dormant (Table 1 , cross 3 )

Among heterozygous seeds, Dfdf (with 'Baroda' background) seed dormancy appeared to be influenced by the maternal parent (Table 1, crosses 15 and 16). Dormancy was broken more rapidly in seeds from a cross where the female was $D f D f$ than for seeds where the female was $d f d f$. Hence, the $d f$ gene may interact with cytoplasmic factors, or there may be a maternal effect in which the offspring phenotype depends on maternal genotype. This effect, however, was not observed with the 'Marketer' background (Table 1, crosses 13 and 14). If, however, the dormancy-inducing factor(s) is caused by a cytoplasmic mechanism or maternal effect that is independent of the $\mathrm{df}$ gene, then crosses with 'Baroda' as the female parent should produce seeds with a higher degree of dormancy. However, in comparing crosses 8 vs. 7,10 vs. 9 , and 12 vs. 11, there was less dormancy when 'Baroda' was the female parent.

From these studies, it is clear that dormancy is not due solely to genotype $d f d f$, and, in fact, the allele has no effect on seed dormancy with some backgrounds. There seems to be an interaction of $d f$ gene with the three genes that actually are responsible for germination (Shifriss and George, 1965). Genotype $d f d f$ induced strong seed dormancy in 'Baroda', and the $D f$ gene could not restore normal germination in this line. In 'Marketer' background, $d f$ gene did not de-

Table 1. Seed germination of various genotypes of 'Baroda' and 'Marketer' cucumber.

\begin{tabular}{|c|c|c|c|c|c|c|c|c|c|}
\hline \multirow{3}{*}{$\begin{array}{l}\text { Cross } \\
\text { no. }\end{array}$} & \multirow{3}{*}{$\begin{array}{l}\text { Parental genotypes } \\
\text { (female } \times \text { male) }\end{array}$} & \multicolumn{8}{|c|}{ Germination $(\%)$} \\
\hline & & \multicolumn{8}{|c|}{ Days after harvest } \\
\hline & & 12 & 25 & 33 & 40 & 59 & 69 & 79 & 85 \\
\hline 1. & MK DfDf self & 100 & & & & & & & \\
\hline 2. & BA dfdf sclf & 0 & 0 & 0 & 0 & 0 & 0 & 0 & $0^{y}$ \\
\hline 3. & MK dfdf self & 100 & & & & & & & \\
\hline 4. & BA DfDf self & 0 & 0 & 0 & 0 & 15 & 30 & 45 & 100 \\
\hline 5. & MK Dfdf $\times$ BA dfdf & 20 & 20 & 60 & 100 & & & & \\
\hline 6. & BA dfdf $\times$ MK DfDf & & & & Unav & able & & & \\
\hline 7. & MK dfdf $\times$ BA DfDf & 0 & 49 & 90 & 100 & & & & \\
\hline 8. & BA DfDf $\times$ MK dfdf & 33 & 100 & & & & & & \\
\hline 9. & MK DfDF $\times$ BA DfDf & 33 & 50 & 50 & 100 & & & & \\
\hline 10. & BA DfDf $\times$ MK DfDf & 100 & & & & & & & \\
\hline 11. & $\mathrm{MK}$ dfdf $\times \mathrm{BA}$ dfdf & 30 & 40 & 70 & 100 & & & & \\
\hline 12. & BA dfdf $\times$ MK dfdf & 85 & 90 & 100 & & & & & \\
\hline 13. & MK DfDf $\times$ MK dfdf & 13 & 100 & & & & & & \\
\hline 14. & MK dfdf $\times$ MK DfDf & 100 & & & & & & & \\
\hline 15. & BA DfDf $\times$ BA dfdf & 0 & 0 & 0 & 15 & 30 & 70 & 100 & \\
\hline 16. & BA dfdf $\times$ BA DfDf & 0 & 0 & 0 & 0 & 0 & 0 & 0 & \\
\hline
\end{tabular}


Table 2. Effect of cotyledon size and inner integument on germination of 'Baroda' $d f d f$ cucumber seed $^{\mathrm{z}}$.

\begin{tabular}{lccr}
\hline & \multicolumn{3}{c}{ Germination (\%) } \\
\cline { 2 - 4 } Seed & \multicolumn{3}{c}{ Days on MS medium } \\
\cline { 2 - 4 } treatment & 5 & 10 & 20 \\
\hline Embryonic axis + no cotyledon & 100 & 100 & 100 \\
Axis $+1 / 4$ cotyledon & 65 & 86 & 86 \\
Axis $+1 / 2$ cotyledon & 50 & 83 & 83 \\
Axis $+3 / 4$ cotyledon & 50 & 83 & 83 \\
Seed with inner integument & 0 & 0 & 0 \\
Seed without inner integument & $\ldots-.-$ & 93 \\
Seed with punctured inner integument & $\ldots$ & $\ldots$ & 91 \\
\hline
\end{tabular}

xPortions of the cotyledons (all, one-fourth, one-half, three-fourths, or none of the cotyledons) were excised from the seeds so that each embryonic axis remained attached to none or some of the original cotyledons.

Table 3. Effect of seed inner integument on water uptake of 'Baroda' $d f d f$ and 'Marketer' DfDf cucumber seed.

\begin{tabular}{|c|c|c|c|c|}
\hline \multirow{3}{*}{$\begin{array}{l}\text { Seed } \\
\text { treatment }\end{array}$} & \multicolumn{4}{|c|}{ Water uptake (mg) ${ }^{\mathbf{z}}$} \\
\hline & \multicolumn{4}{|c|}{ Time on MS medium (h) } \\
\hline & 0 & 6 & 12 & 24 \\
\hline \multicolumn{5}{|l|}{ Baroda $d f d f$} \\
\hline With inner integument & $1.3 \mathrm{a}$ & $9.0 \mathrm{~b}$ & $10.9 \mathrm{~b}$ & $9.5 \mathrm{c}$ \\
\hline Inner integument punctured & $1.3 \mathrm{a}$ & $9.0 \mathrm{~b}$ & $12.8 \mathrm{a}$ & $12.6 \mathrm{~b}$ \\
\hline \multicolumn{5}{|l|}{ Marketer DfDf } \\
\hline With inner integument & $1.3 \mathrm{a}$ & $9.5 \mathrm{~b}$ & $9.8 \mathrm{~b}$ & $10.5 \mathrm{c}$ \\
\hline Inner integument punctured & $1.3 \mathrm{a}$ & 12.1. a & $13.3 \mathrm{a}$ & $14.2 \mathrm{a}$ \\
\hline
\end{tabular}

lay seed germination. Therefore, it is likely that dormancy is caused by other factors and the $D f$ gene reduces the duration of dormancy. Possibly some modifier gene(s) is involved. These modifiers seem to have stronger effect in the presence of $d f$ gene, and their interaction with genotype $D f D f$ may not be so strong. In the present study, BA $d f d f$ seeds remained dormant for $>144$ days.

To determine whether a particular portion of the 'Baroda' $d f d f$ seed was responsible for delayed germination, seeds were washed with $0.5 \%$ sodium hypochlorite for $10 \mathrm{~min}$, rinsed with water, and the seed coat was removed. Portions of the cotyledons (zero, one-fourth, one-half, three-fourths, or all of the cotyledons) were excised from 20 seeds so that each embryonic axis remained attached to none or some of the original cotyledons. To determine if the inner integument was involved in dormancy, lots of 15 seeds each were subjected to one of three treatments. The inner integument was removed entirely from one lot of seeds, while a second lot had the inner integument punctured with a needle. These were compared with 15 seeds that had only the seed coat removed, leaving the integument intact. These were placed on $\mathrm{Mu}-$ rashige and Skoog (1962) medium (MS) without growth regulators. Germination was monitored for 20 days.

To determine if the inner integument was limiting gas exchange or water uptake, seeds were disinfected and rinsed as above and the seed coat was removed. Thirty seeds of 'Baroda' $d f d f$ and 'Marketer' $D f D f$ had the inner integument punctured and were compared with 30 seeds with only the seed coat removed. Seeds were placed on MS medium containing no hormones. After $0,6,12$, or $24 \mathrm{~h}$, seeds were blotted to absorb free moisture, weighed, and dried at $60 \mathrm{C}$ for $48 \mathrm{~h}$. Water uptake by individual seeds was calculated. Data were analyzed by a general linear regression model.

Removing part or all of the cotyledons dramatically increased germination of 'Baroda' $d f d f$ (Table 2). The embryonic axis alone germinated in 5 days, while removal of part of the cotyledons increased germination to $83 \%$ within 10 days. When the inner integument was removed, 93\% of the 'Baroda' $d f d f$ seeds germinated in 20 days. Puncturing the inner integument also broke the dormancy of 'Baroda' $d f d f$ (Table 2).

For all treatments, seed water content reached a plateau after $12 \mathrm{~h}$ of imbibition, with little or no additional water uptake occurring between 12 and $24 \mathrm{~h}$ (Table 3). Within a cultivar, there were significant differences in water uptake between punctured and unpunctured seeds after $12 \mathrm{~h}$. However, seeds at this hydration level usually germinated. At all times, there was no difference between water absorption of BA and MK seeds with the inner integument intact (Table 3); yet the MK seeds germinated and the BA seeds did not (Table 1). Therefore, dormancy was not induced by a lack of water absorption.

Any treatment (puncturing the inner integument, its complete removal, or partial removal with the cotyledons) that damaged the inner integument allowed BA seed to germinate. This pattern suggests that the intact inner integument is responsible for maintaining dormancy of BA seeds, possibly because it acted as a physical barrier to gas exchange or impeded the outward flow of some dormancy-inducing chemical(s) produced by the embryonic axis or cotyledons. Takahashi and Suge (1972) reported ethylene production by cucumber cotyledons, which is known to inhibit seed germination in some crops (Evenari, 1949). If the inner integument of the 'Baroda' $d f d f$ seeds were impermeable to gas exchange, endogenous increases in ethylene concentration within the seed might inhibit germination and induce dormancy.

\section{Literature Cited}

Evenari, M. 1949. Germination inhibitors. Bot. Rev. 15:153-194.

Murashige, T. and F. Skoog. 1962. A revised medium for rapid growth and bioassays with tobacco tissue cultures. Physiol. Plant 15:474497.

Shifriss, O. and W.L. George. 1965. Delayed germination and flowering in cucumber. Nature (London) 206:424-425.

Takahashi, H. and H. Suge. 1972. Sex expression and ethylene production in cucumber plants as affected by 1-aminocyclopropane-1-carboxylic acid. J. Jpn. Soc. Hort. Sci. 51:51-55. 